$10^{5}$ copies/ml compared to culture-negative: $2.7 \times 10^{5}$ copies/ ml samples $(p=0.499)$.

Discussion/conclusion The gonococcal bacterial loads were similar between saliva and the pharynx and not influenced by culture status. Saliva could be important in the transmission of gonorrhoea such as oral-anal sex and saliva use as a lubricant for anal sex.

\section{P090 ASSORTATIVE SEXUAL MIXING PATTERNS IN MALE- FEMALE AND MALE-MALE PARTNERSHIPS IN MELBOURNE, AUSTRALIA: IMPLICATIONS FOR HIV AND STI TRANSMISSION}

\begin{abstract}
1,2 Eric Chow* $^{*}{ }^{1,2} \mathrm{Tim}$ Read, ${ }^{3}$ Matthew Law, ${ }^{1,2}$ Marcus Chen, 1,2 Catriona Bradshaw, ${ }^{1,2}$ Christopher Fairley. 'Melbourne Sexual Health Centre, Alfred Health, Melbourne, VIC, Australia; ${ }^{2}$ Central Clinical School, Faculty of Medicine, Nursing and Health Sciences, Monash University, Melbourne, VIC, Australia; ${ }^{3}$ The Kirby Institute, UNSW Australia, UNSW Australia, Australia
\end{abstract}

\subsection{6/sextrans-2016-052718.144}

Background/introduction Assortative (like-with-like) mixing pattern has become a new and important focus in HIV/STI research in recent years in order to understand the mixed sexual network. There are very limited data on sexual mixing patterns, particularly in an Australian population.

Aim(s)/objectives To understand the assortative sexual mixing patterns for age, number of partners, and condom use in malefemale and male-male partnerships in Melbourne between 2011 and 2014.

Methods 1165 male-female and 610 male-male partnerships were included. Correlation between age of partners was examined by the Spearman's rank correlation. The Newman's assortativity coefficient was used as an aggregate quantitative measurement of sexual mixing of number of partners and condom use.

Results There was a strong positive correlation between age of partners in both male-female (rho $=0.709 ; p<0.001$ ), and male-male partnerships (rho $=0.553 ; p<0.001$ ). The assortative mixing pattern for number of partners was similar in malefemale $(r=0.255)$, and male-male partnerships $(r=0.264)$. This pattern decreased over time in male-male $(\mathrm{p}=0.034)$ but not in male-female ( $p=0.718)$ partnerships. There was a stronger assortative mixing pattern for condom use in male-male $(r=0.517)$ compared to male-female $(r=0.382)$ partnerships.

Discussion/conclusion Male-female and male-male partnerships have a high assortativity mixing patterns with respects for age, number of partners, and condom use. Individuals are more likely to connect with partners with of similar age and sexual experience. The sexual mixing pattern is not purely assortative; and hence it may lead to increased HIV and STI transmission in certain risk groups.

\section{P091 EVALUATION OF THE CURRENT AND PROSPECTIVE ROLE OF POOLED SAMPLING FOR SEXUALLY TRANSMITTED INFECTION TESTING: A WEB-BASED SURVEY OF GENITOURINARY MEDICINE SERVICES IN ENGLAND}

Jonathan Shaw*, John Saunders, Gwenda Hughes. Public Health England, London, UK
Introduction Chlamydia trachomatis and Neisseria gonorrhoeae testing guidance recommends extragenital screening with locally validated nucleic acid amplification tests for patients reporting receptive oral and/or anal sex, with anatomical sites sampled and tested separately. Within-patient pooled sampling (PS) could be more cost effective for triple -site (genital/oral/anal) testing, but may require establishment of complex management pathways and loss of information to guide risk assessments and treatment.

Objectives We reviewed the evidence on the cost effectiveness of PS and explored current opinion and practice among genitourinary medicine (GUM) clinics in England.

Methods Global literature on PS was reviewed. A web-based survey was distributed to GUM clinical leads throughout England on $11 / 02 / 16$.

Results Published evidence supports multi-patient combined aliquot PS for population screening, however evidence for within-patient PS is sparse. 44/223 (19.7\%) services responded to the web survey. One service $(2.3 \%)$ reported current PS and 2 $(4.5 \%)$ were awaiting implementation. Of the 41 services not pooling, 4 (9.8\%) were considering future implementation. Commonly reported barriers to implementation of PS were: loss of infection site information (30/44, 68.2\%), absence of national guidance $(26 / 44,59.1 \%)$, and decreases in assay sensitivity/specificity (17/44, 38.6\%). Only 6/44 (13.6\%) considered the current level of evidence sufficient to support PS, with $35 / 44$ $(79.5 \%)$ requesting further validation studies, 34/44 (77.3\%) national guidance, and 23/44 (52.3\%) more cost effectiveness data.

Conclusion PS is currently uncommon in GUM services across England. Best practice evidence-based guidance on the appropriate use of PS will be needed if PS is introduced more widely as part of cost-saving measures.

\section{P092 PELVIC INFLAMMATORY DISEASE: A REVIEW OF PRESENTATIONS TO OUR SERVICE}

Frances Hunt, Kirsten Michie*. Royal Liverpool Hospital, Liverpool, UK

\subsection{6/sextrans-2016-052718.146}

Background/introduction Pelvic inflammatory disease (PID) is an important complication of the sexually transmitted infections Chlamydia trachomatis and Neisseria gonorrhoea.

Aim(s)/objectives We sought to review the presentation and management of women treated for PID attending our service.

Methods We used the SHHAPT code C5A to identify women diagnosed with PID between 01/06/2015-30/11/2015. We performed a retrospective case note review of all women, collecting demographic data and details of their presentation and management.

Results 50 cases were identified. The women ranged from ages 17-40 years, median 23.5 years. Presenting complaints were pelvic pain (38/50), discharge (21/50), dyspareunia (14/50) and bleeding irregularities (14/50). The majority of women $(40 / 50)$ reported having a regular male partner, and most $(42 / 50)$ had had one partner only in the preceding 3 months. $4 / 50(8.0 \%)$ women tested positive for chlamydia, all of whom were aged less than 25 years. No other sexually transmitted infections were identified. The rate of chlamydia amongst women less than 25 treated for PID was 4/31 (12.9\%). Less than half of women (19/ 50 ) attended for follow up, and there was documentation of the regular partner attending for treatment in only 14/40 cases. 
Discussion/conclusion Our project reflects Chlamydia as an important cause of PID in younger women. This supports the latest guidance recommending repeat Chlamydia screening in under 25 s to identify reinfections and reduce the risk of complications such as PID.

\section{P093 AUDIT ON THE MANAGEMENT OF EPIDIDYMO-ORCHITIS IN A LONDON-BASED LEVEL 3 SEXUAL HEALTH CLINIC}

Temi Lampejo* ${ }^{*}$ Mohamed Abdulcadir. Chelsea \& Westminster Hospital, London, UK

\subsection{6/sextrans-2016-052718.147}

Background/introduction The aetiology of epididymo-orchitis is largely related to a patient's age with sexually transmitted pathogens being the common aetiological agents in those under 35 years of age. In individuals aged over 35 uropathogens represent the commonest cause. National guidelines exist for the appropriate management of this condition.

Aim(s)/objectives To assess the management of epididymo-orchitis in our clinic with reference to the BASHH guidelines.

Methods A case note review of all men with epididymo-orchitis attending our clinic between January and June 2015. Age at time of diagnosis, investigations and treatment decisions were recorded.

Results A total of 59 patients were identified ranging from age 16 to 67 . Only $66 \%$ of patients had all four recommended microbiological investigations performed (target 90\%). Nineteen patients did not have an MSU microscopy/culture performed and 7 had no urethral smear. All patients were tested for chlamydia and gonorrhoea. All 59 patients were prescribed an appropriate antibiotic regimen. The 5 patients who did not respond clinically had a documented plan for further clinical action.

Abstract P093 Table 1 Epididymo-orchitis

\begin{tabular}{llll}
\hline & Age $\leq 35$ years & Age $>35$ years & Total \\
\hline No. of patients & 38 & 21 & 59 \\
CT positive & 5 & 0 & 5 \\
GC positive & 0 & 0 & 0 \\
MSU positive & 1 & 1 & 2 \\
\hline
\end{tabular}

Discussion/conclusions This audit demonstrated that patients attending our clinic were treated in concordance with national guidelines and the vast majority showed a good clinical response. However, lack of routine urine sampling for microscopy/culture was evident. Although a urine dipstick was performed in most cases, guidelines do stipulate that this only serves as a useful adjunct. As a result of this audit our department intends to obtain an MSU for culture in all cases of epididymo-orchitis.

\section{P094 CHLAMYDIA TRACHOMATIS (CT) POSITIVITY RATE AT 2 WEEK NEISSERIA GONORRHOEAE (NG) TEST OF CURE (TOC)}

Nicolo Girometti, Joseph Scott, Kimberly Rhodes, Alan McOwan*, Nneka Nwokolo, Gary Whitlock. ${ }^{56}$ Dean Street, Chelsea \& Westminster Hospital, London, UK
Background/introduction Whilst guidelines recommend NG TOC 2 weeks after treatment, there is little data on the optimum time to perform a TOC for CT in those for whom this is indicated. Current BASHH guidelines recommend deferring TOC for at least 3 weeks after treatment because residual chlamydial DNA may persist.

Aim(s)/objectives Patients who are treated for NG and CT coinfection re-attending for subsequent NG TOC are tested for both infections by NAAT providing the opportunity to evaluate the CT positivity rate at re-attendance.

Methods A retrospective case review of co-infected GC/CT positive (analysed with Cepheid GeneXpert) patients tested in a London sexual health clinic over 12 consecutive months was performed. TOC details were evaluated, and appropriate antibiotic treatment according to $\mathrm{BASHH}$ guidelines was assessed.

Results 480 patients tested positive for both infections and 132 attended for TOC within 21 days of treatment (median 15 days, IQR 14-17). Of these 131 were male, of whom 126 MSM; median age was $35 \mathrm{y}$ and median number of sexual partners in previous 3 months was 5. Site of CT infection was rectal (94), urethral (49), throat (11), vulvovaginal (1). At TOC, 6 (4.5\%) had a persistent positive CT NAAT: rectum (3), urethra (3). One patient with persistent rectal CT had received treatment with azithromycin; the other 5 received BASHH preferred treatment. By comparison, $3(2.3 \%)$ had a positive NG NAAT at TOC.

Discussion CT positivity 15 days after treatment is low, suggesting that TOC at 2 weeks may be a possible management strategy.

\section{P095 GETTING HIGH AND HAVING SEX- ARE YOUNG WOMEN JOINING THE PARTY?}

Nicky Dearing*, Sophie Herbert. Nottingham University Hospitals NHS Trust, Nottingham, UK

\subsection{6/sextrans-2016-052718.149}

Background Use of 'chems' by MSM (men who have sex with men) is reported widely and is associated with poor sexual health outcomes but less is known for the general GU clinic population.

Aims To determine the proportion of men and women reporting recreational drug use and identify sexual risk taking and health outcomes.

Methods Patients attending GUM from 1-21 ${ }^{\text {st }}$ December 2015 were invited to complete an anonymous paper questionnaire. Age, sexual orientation, sexual partners, STIs, smoking, drug and alcohol use were collected.

Results 128 men (32.8\% MSM) and 101 women responded. 19\% women, 36\% heterosexual men (HM) and 52\% MSM reported recreational drug use in the past 12 months. Women users were younger (age range 19-42, median 23) and their preferred drug was Cocaine (12\%). Men were older (age range 1967, median 28), cocaine was a preferred drug (28\% HM, 19\% MSM) but MSM also used Mephadrone, Ecstasy and Viagra equally (19\%). Users reported UPSI with multiple partners in the last 3 months more often (68\% MSM, 50\% HM, 53\% females) compared with non users (30\% MSM, 26\% HM and 17\% females). Female users reported the highest recent STI rates, 68\% (MSM 55\%) and non-consensual sex (21\%).

Discussion We found significant drug use and risky sexual behaviour amongst heterosexuals, although MSM remain the highest 